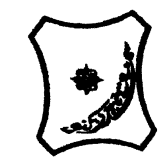

Bayero Journal of Pure and Applied Sciences, 10(1): 227 - 233

Received: June, 2016

Accepted: January, 2017

ISSN $2006-6996$

\title{
PREVALENCE OF PATHOGENIC MICROORGANISMS IN THE ORAL CAVITY AND THEIR SENSITIVITY TO SOME SELECTED DENTIFRICE SOLD AT MAJOR MARKETS IN KANO METROPOLIS
}

\begin{abstract}
${ }^{* 1}$ Yahaya S. and ${ }^{1}$ Ramatu, A. M.
${ }^{1}$ Department of Microbiology, Faculty of Science, Bayero University, Kano.

*Corresponding author: syahaya.mcb@buk.edu.ng; sanishinkafi@gmail.com, Mobile: +234 (0) 8065168080

\section{ABSTRACT}

This study was conducted to determine the incidence of pathogenic microorganisms associated with dental caries and antimicrobial susceptibility test of some common dentifrice sold in Kano metropolis. A total of 50 samples were used in this study. The samples were taken using swab from human oral mucosa. The swabs were inoculated on chocolate agar, blood agar and MacConkey agar incubated at $37^{\circ} \mathrm{Cfor} 24 \mathrm{~h}$, as well as Sabouraud's dextrose agar incubated at room temperature for 48h. Dentifrices were also analyzed for the fluoride, phosphate, nitrite, potassium, chloride, carbonate, sulphate, calcium, sodium, and zinc contents using C: Varian/Cary Winuv method, Acid Base Titration method, Gravimetry, and Air/Acetylene method. Of all the isolates, Streptococcus mutans was the most isolated pathogen with 24(30.38\%), followed by Staphylococcus aureus with 23(29.11\%), Candida. albicans 14(17.72\%), Klebsiella specie 10(12.66\%) and Escherichia coli 8(10.13\%). Disc diffusion method with ten different dentifrices (code A-J) was tested for their antimicrobial activity against isolated oral pathogens. The samples were tested in triplicate, at full strength, 1:1, 1:2, 1:4, 1:8 and 1:16 dilutions. Inhibition zones were measured in millimeter after 24 hours. All the tested dentifrices demonstrated an antimicrobial activity. Dentifrice $A$ showed the maximum mean zones of inhibition of 26.67, 24.00, 22.27, 19.67 and 18.67 against Klebsiella specie, Escherichia coli, Staphylococcus aureus, Streptococcus mutans and Candida albicans respectively, while dentifrice $J$ showed the least activity of $0.00,0.00,0.00,14.67$ and 18.67 respectively against the same organisms. The concentrations of fluoride range between 8.3$132.3 \mathrm{mg} / \mathrm{L}$; phosphate, $45.8-191.8 \mathrm{mg} / \mathrm{L} ;$ nitrite, $11.0 \mathrm{mg} / \mathrm{L} ;$ potassium, $3.2 \mathrm{mg} / \mathrm{L}$; chloride, 5.1 $5.6 \mathrm{mg} / \mathrm{L}$; carbonate, $8.2-12.8 \mathrm{mg} / \mathrm{L}$; sulphate, 0.3-0.9g; calcium, 14.7-16.1mg/L; sodium, 350.6$418.8 \mathrm{mg} / \mathrm{L}$ and zinc, $6.2 \mathrm{mg} / \mathrm{L}$. In the present study, it has been demonstrated that triclosancontaining dentifrice are more effective in the control of pathogenic microorganisms associated with oral cavity compared to non-triclosan containing dentifrice.

Keywords: Bacteria, Dentifrice, Kano, Oral cavity.
\end{abstract}

\section{INTRODUCTION}

Dentifrice is a paste or gel used with a toothbrush as an accessory to clean and maintain the aesthetics and health of teeth. Dentifrice is used to promote oral hygiene, it serves as an abrasive that aids in removing the dental plaque and food from the teeth, assists in suppressing halitosis, and delivers active ingredients (most commonly fluoride) to help prevent tooth and gum disease called gingivitis (American Dental Association, 2010). A very significant proportion of dental problem are due to microbial infection. Dental problems are of three types, formation of dental plaque, dental caries and periodontal disease (Clarke, 1924). Plaque is a layer that forms on the surface of a tooth that has been linked to gingivitis, periodontal disease or dental caries (Jensena and Barkvoll, 1998). Periodontal diseases are bacterial infections that affect the supporting structure of the teeth (gingival, cementum, periodontal membrane and alveolar bone). Serious forms of periodontal disease that affect the periodontal membrane and alveolar bone may results in tooth loss. Streptococci, Spirochetes and Bacteroides are found to be the possible pathogens responsible for the disease (Manupati, 2010). Dental caries is a colonized, transmissible infectious process that ends up in the destruction of hard dental tissue. Streptococcus mutans is one of the main opportunistic pathogens of dental caries (Gamboa et al., 2004). In addition, other microflora like Escherichia coli and Candida albicans are also associated with active caries lesion (Oztan et al., 2006). Some constituents of tooth paste (fluoride, phosphate, nitrite, potassium, chlouride, sulphate, calcium, sodium, and magnesium) are of great importance in physiological functioning and development of human body. Such constituent may pass in to human body directly or indirectly during mouth washing. Since tooth paste are household essentials and many brand flood the market, their nature and form of usage is a subject of research interest (Oyewale, 2005). This study aimed to assess the prevalence of pathogenic microorganisms in the oral cavity and their sensitivity to some selected dentifrices sold at some major markets in Kano metropolis. 
MATERIAL AND METHODS

\section{Sample Collection and processing}

Oral samples were collected after explaining the procedure to the consented persons. For each individual both sides of buccal mucosa were gently rubbed with a sterile cotton swab for 15 seconds. The swab were properly labelled and transported in a safety box within 1 hour to the laboratory for isolation. Microbiological analyses

The specimens were inoculated onto Nutrient agar supplemented with $5 \%$ sheep blood when the agar base cooled to $56^{\circ} \mathrm{C}$ (Chocolate agar) and $50^{\circ} \mathrm{C}$ (Blood agar) for the isolation of Streptococcus mutans and Staphylococcus aureus respectively. The plates were then incubated at $37^{\circ} \mathrm{C}$ for 24 hour in candle jar. $S$. mutans and $S$. aureus colonies were chosen based on their morphology and further confirmation was done using biochemical tests such as Catalase and Coagulase test as described by Cheesbrough (2004) and optochin sensitive test (for $S$. mutans) by Patterson (1996). MacConkey agar was inoculated for the isolation of Gram negative bacteria and the plates were incubated at $37^{\circ} \mathrm{C}$ for 24 hour aerobically and further confirmed by urease test, sugar fermentation test, citrate utilization test and indole test (Cheesbrough, 2004). Candida albicans was isolated on Sabouraud's Dextrose agar at room temperature for 48 hour and further confirmed by Germ tube test as described by Cheesbrough (2004).

\section{Antimicrobial Assessment of the sampled Dentifrices}

A total of ten (10) different types of dentifrice were used in this study. Dentifrice was prepared in sterile distilled water $(1 \mathrm{~g} / \mathrm{ml})$. Sterile filter disc were impregnated with the $50 \mathrm{~g}$ of dentifrice as full strength and some sterile filter discs soaked in $1: 1,1: 2,1: 4$,
$1: 8$ and $1: 16$ dilutions. Then the discs were dried in an oven at $60^{\circ} \mathrm{C}$ for 2 hours (Sadeghi and Assar, 2009). Antimicrobial activities were determined by disc diffusion (Kirby-Baeur) method. Mueller Hinton agar was used to demonstrate the antimicrobial effect on Escherichia coli, Klebsiella spp. and Staphylococcus aureus, while Brain Heart Infusion agar was used for Streptococcus mutans and Sabouraud's Dextrose agar for Candida albicans. Afterwards, the plates were incubated at $37^{\circ} \mathrm{C}$ for 24 hours (48 hours for yeast).

Elemental Analysis of the dentifrices

In all the samples $10 \%$ solution of the dentifrice were used for the analysis. Concentration of fluoride, phosphate, nitrite, potassium, chloride, calcium, and sodium were determined using C: Varian/Cary Winuv method with Carry 50 instrument. Concentration of carbonate was determined using Acid Base Titration method, concentration of sulphate was determined using Gravimetric method with filter paper and concentration of zinc was determined using Air/Acetylene method with AAS Buck Scientific 210 VGP.

\section{Statistical Analysis}

Percentage was used for the number of isolates. The mean values of inhibitory zones for various dentifrices were analyzed by one-way analysis of variance (ANOVA) using Sigma Plot Statistical software. Values were considered significant when $\mathrm{p}<0.05$.

\section{RESULTS}

Sixty five colonies of bacteria and fourteen of $C$. albicans were isolated from the fifty specimens analyzed in this work (Table 1). S. mutans had the highest frequency of 24 colonies $(30.38 \%)$ while $E$. coli had the least frequency of 8 colonies $(10.13 \%)$.

\begin{tabular}{lcc} 
Table 1: Distribution of isolates and their percentages & \\
\hline Organisms & Frequency of Isolates & \% Frequency \\
\hline S. Mutans & 24 & 30.38 \\
S. aureus & 23 & 29.11 \\
K. specie & 10 & 12.66 \\
E. coli & 8 & 10.13 \\
C. albicans & 14 & 17.72 \\
TOTAL & $\mathbf{7 9}$ & $\mathbf{1 0 0}$ \\
\hline
\end{tabular}

Results for antimicrobial activity of the 10 dentifrice formulations against $S$. aureus indicated that dentifrice designated as ' $A$ ' produced the highest mean zone diameter of inhibition of $22.27 \pm 1.53 \mathrm{~mm}$ using full strength and $11.33 \pm 2.08 \mathrm{~mm}$ at $1: 16$ dilution. Dentifrice $\mathrm{G}, \mathrm{H}$, and J produced no zone diameter of inhibition at all the concentrations tested (Table 2). The antimicrobial activity exhibited by these dentifrices on $S$. mutans indicates that dentifrice A had the highest effect while dentifrice $\mathrm{J}$ had the least effect (Table 2). A mean diameter zone of inhibition of $19.67 \pm 1.53 \mathrm{~mm}$ and $9.67 \pm 0.58 \mathrm{~mm}$ were produced by full strength and 1:16 dilution formulation of $\mathrm{A}$ dentifrice (Table 3). All the 10 dentifrices were found to demonstrate zone diameter of inhibition at least using the $1: 1$ dilution. The results for antimicrobial activity of the 10 tested dentifrices against Klebsiella. species were shown in Table 4. Dentifrice formulation $A$ had the maximum mean zone diameter of inhibition of $26.67 \pm 1.53 \mathrm{~mm}$ and $14.33 \pm 1.15 \mathrm{~mm}$ using fill strength and 1:16 dilution. Only four dentifrices were found to produce antimicrobial activity against Klebsiella. species. Dentifrice E, F, G, H, I and J produced no zone diameter of inhibition at all the concentrations tested. Results for antimicrobial activity of the tested dentifrices formulations against E. coli were shown in Table 5. Dentifrice formulation A exhibited the highest mean zone diameter of inhibition of $24.00 \pm 1.00 \mathrm{~mm}$ using full strength and $13.00 \pm 2.00 \mathrm{~mm}$ using $1: 16$ dilution. While dentifrice I and $\mathrm{J}$ produced no zone diameter of inhibition at all the concentrations tested dentifrice. The antimicrobial activity exhibited by 10 dentifrices on $C$. albicans indicates that dentifrice formulation $\mathrm{F}$ had the highest mean zone diameter of inhibition of $22.00 \pm 1.73 \mathrm{~mm}$ and $10.00 \pm 2.00 \mathrm{~mm}$ were produced by full strength and 1:8 dilutions. Dentifrice formulation I had the least activity of zero zone diameter of inhibition at all the concentration. 
Bajopas Volume 10 Number 1 June, 2017

Table 2: The Mean Diameter of Inhibition Zones $(\mathrm{mm})$ of Anti-microbial Activity of Dentifrice Formulations against Staphylococcus aureus

\begin{tabular}{|c|c|c|c|c|c|c|}
\hline $\begin{array}{l}\text { Dentifrice } \\
\text { Furmulation }\end{array}$ & $\mathrm{FS}$ & 1:1 Dilution & 1:2 Dilution & 1:4 Dilution & 1:8Dilution & 1:16 Dilution \\
\hline $\bar{A}$ & $22.27 \pm 1.53$ & $19.67 \pm 2.08$ & $17.00 \pm 1.73$ & $16.67 \pm 1.15$ & $14.67 \pm 0.58$ & $11.33 \pm 2.08$ \\
\hline B & $21.00 \pm 1.73$ & $19.33 \pm 1.53$ & $16.00 \pm 2.00$ & $0.00 \pm 0.00$ & $0.00 \pm 0.00$ & $0.00 \pm 0.00$ \\
\hline C & $20.00 \pm 1.00$ & $17.67 \pm 1.15$ & $16.33 \pm 1.53$ & $12.67 \pm 1.15$ & $12.33 \pm 1.53$ & $9.67 \pm 2.08$ \\
\hline D & $18.67 \pm 1.53$ & $17.00 \pm 1.00$ & $15.33 \pm 0.58$ & $0.00 \pm 0.00$ & $0.00 \pm 0.00$ & $0.00 \pm 0.00$ \\
\hline$E$ & $19.33 \pm 2.08$ & $17.00 \pm 2.00$ & $16.00 \pm 2.67$ & $14.33 \pm 2.52$ & $11.67 \pm 2.89$ & $0.00 \pm 0.00$ \\
\hline $\mathrm{F}$ & $20.67 \pm 1.53$ & $14.33 \pm 1.15$ & $12.33 \pm 1.15$ & $10.67 \pm 0.58$ & $0.00 \pm 0.00$ & $0.00 \pm 0.00$ \\
\hline G & $0.00 \pm 0.00$ & $0.00 \pm 0.00$ & $0.00 \pm 0.00$ & $0.00 \pm 0.00$ & $0.00 \pm 0.00$ & $0.00 \pm 0.00$ \\
\hline $\mathrm{H}$ & $0.00 \pm 0.00$ & $0.00 \pm 0.00$ & $0.00 \pm 0.00$ & $0.00 \pm 0.00$ & $0.00 \pm 0.00$ & $0.00 \pm 0.00$ \\
\hline I & $11.67 \pm 0.58$ & $10.00 \pm 0.00$ & $9.67 \pm 1.15$ & $8.00 \pm 1.00$ & $0.00 \pm .000$ & $0.00 \pm 0.00$ \\
\hline J & $0.00 \pm 0.00$ & $0.00 \pm 0.00$ & $0.00 \pm 0.00$ & $0.00 \pm 0.00$ & $0.00 \pm 0.00$ & $0.00 \pm 0.00$ \\
\hline Control & $0.00 \pm 0.00$ & $0.00 \pm 0.00$ & $0.00 \pm 0.00$ & $0.00 \pm 0.00$ & $0.00 \pm 0.00$ & $0.00 \pm 0.00$ \\
\hline
\end{tabular}

FS: Full strength. $\quad$ No significant difference: $(P=<0.001)$.

Control: Sterile filter disc.

Table 3: Table 3. The Mean Diameter of Inhibition Zones $(\mathrm{mm})$ of Anti-microbil Activity of Dentifrice Formulations against Streptococcus mutans

\begin{tabular}{lllllll}
\hline Dentifrice Formulation & FS & $1: 1$ Dilution & $1: 2$ Dilution & $1: 4$ Dilution & $1: 8$ Dilution & $1: 16$ Dilution \\
& & & & & & \\
\hline A & $19.67 \pm 1.53$ & $18.33 \pm 1.15$ & $15.33 \pm 1.15$ & $12.33 \pm 1.53$ & $12.00 \pm 1.00$ & $9.67 \pm 0.58$ \\
B & $15.67 \pm 1.15$ & $13.33 \pm 1.53$ & $10.67 \pm 0.58$ & $8.00 \pm 2.00$ & $0.00 \pm 0.00$ & $0.00 \pm 0.00$ \\
C & $10.00 \pm 1.73$ & $8.00 \pm 1.00$ & $5.33 \pm 1.15$ & $0.00 \pm 0.00$ & $0.00 \pm 0.00$ & $0.00 \pm 0.00$ \\
D & $10.00 \pm 2.00$ & $5.67 \pm 1.15$ & $0.00 \pm 0.00$ & $0.00 \pm 0.00$ & $0.00 \pm 0.00$ & $0.00 \pm 0.00$ \\
E & $16.67 \pm 2.08$ & $14.67 \pm 2.89$ & $11.33 \pm 0.58$ & $7.33 \pm 1.53$ & $0.00 \pm 0.00$ & $0.00 \pm 0.00$ \\
F & $11.67 \pm 1.53$ & $7.67 \pm 2.52$ & $0.00 \pm 0.00$ & $0.00 \pm 0.00$ & $0.00 \pm 0.00$ & $0.00 \pm 0.00$ \\
G & $11.33 \pm 0.58$ & $9.00 \pm 1.00$ & $0.00 \pm 0.00$ & $0.00 \pm 0.00$ & $0.00 \pm 0.00$ & $0.00 \pm 0.00$ \\
H & $10.67 \pm 1.53$ & $8.67 \pm 1.15$ & $7.00 \pm 2.00$ & $0.00 \pm 0.00$ & $0.00 \pm 0.00$ & $0.00 \pm 0.00$ \\
I & $12.33 \pm 2.52$ & $10.67 \pm 2.08$ & $8.00 \pm 2.67$ & $0.00 \pm 0.00$ & $0.00 \pm 0.00$ & $0.00 \pm 0.00$ \\
J & $14.67 \pm 1.15$ & $11.33 \pm 0.58$ & $8.67 \pm 1.53$ & $6.67 \pm 2.08$ & $0.00 \pm 0.00$ & $0.00 \pm 0.00$ \\
Control & $0.00 \pm 0.00$ & $0.00 \pm 0.00$ & $0.00 \pm 0.00$ & $0.00 \pm 0.00$ & $0.00 \pm 0.00$ & $0.00 \pm 0.00$
\end{tabular}

FS: Full strength. No significant difference: $(P=<0.001)$.

Control: Sterile filter disc.

Table 4: The Mean Diameter of Inhibition Zones $(\mathrm{mm})$ of Anti-microbil Activity of Dentifrice Formulations against Klebsiella species

\begin{tabular}{lllllll}
$\begin{array}{l}\text { Dentifrice } \\
\text { Formulation }\end{array}$ & FS & $1: 1$ Dilution & $1: 2$ Dilution & $1: 4$ Dilution & $1: 8$ Dilution & $1: 16$ Dilution \\
\hline A & $26.67 \pm 1.53$ & $24.33 \pm 0.58$ & $22.00 \pm 1.00$ & $20.00 \pm 0.00$ & $18.00 \pm 1.00$ & $14.33 \pm 1.15$ \\
B & $17.00 \pm 1.73$ & $14.00 \pm 1.00$ & $11.33 \pm 1.15$ & $7.67 \pm 0.58$ & $6.33 \pm 1.15$ & $0.00 \pm 0.00$ \\
C & $17.33 \pm 1.73$ & $14.33 \pm 0.58$ & $12.00 \pm 1.00$ & $9.67 \pm 0.58$ & $8.33 \pm 1.15$ & $6.33 \pm 1.15$ \\
D & $16.67 \pm 0.58$ & $14.00 \pm 1.00$ & $10.67 \pm 1.53$ & $9.33 \pm 1.15$ & $6.67 \pm 0.58$ & $0.00 \pm 0.00$ \\
E & $0.00 \pm 0.00$ & $0.00 \pm 0.00$ & $0.00 \pm 0.00$ & $0.00 \pm 0.00$ & $0.00 \pm 0.00$ & $0.00 \pm 0.00$ \\
F & $0.00 \pm 0.00$ & $0.00 \pm 0.00$ & $0.00 \pm 0.00$ & $0.00 \pm 0.00$ & $0.00 \pm 0.00$ & $0.00 \pm 0.00$ \\
G & $0.00 \pm 0.00$ & $0.00 \pm 0.00$ & $0.00 \pm 0.00$ & $0.00 \pm 0.00$ & $0.00 \pm 0.00$ & $0.00 \pm 0.00$ \\
H & $0.00 \pm 0.00$ & $0.00 \pm 0.00$ & $0.00 \pm 0.00$ & $0.00 \pm 0.00$ & $0.00 \pm 0.00$ & $0.00 \pm 0.00$ \\
I & $0.00 \pm 0.00$ & $0.00 \pm 0.00$ & $0.00 \pm 0.00$ & $0.00 \pm 0.00$ & $0.00 \pm 0.00$ & $0.00 \pm 0.00$ \\
J & $0.00 \pm 0.00$ & $0.00 \pm 0.00$ & $0.00 \pm 0.00$ & $0.00 \pm 0.00$ & $0.00 \pm 0.00$ & $0.00 \pm 0.00$ \\
Control & $0.00 \pm 0.00$ & $0.00 \pm 0.00$ & $0.00 \pm 0.00$ & $0.00 \pm 0.00$ & $0.00 \pm 0.00$ & $0.00 \pm 0.00$ \\
\hline
\end{tabular}

FS: Full strength. No significant difference: $(P=<0.001)$.

Control: Sterile filter disc. 
Bajopas Volume 10 Number 1 June, 2017

Table 5: The Mean Diameter of Inhibition Zones $(\mathrm{mm})$ of Anti-microbil Activity of Dentifrice Formulations against Escherichia coli

\begin{tabular}{lllllll}
\hline $\begin{array}{l}\text { Dentifrice } \\
\text { Formulation }\end{array}$ & FS & $1: 1$ Dilution & $1: 2$ Dilution & $1: 4$ Dilution & $1: 8$ Dilution & $1: 16$ Dilution \\
\hline A & $24.00 \pm 1.00$ & $21.67 \pm 1.53$ & $19.67 \pm 2.08$ & $18.00 \pm 1.73$ & $16.33 \pm 0.58$ & $13.00 \pm 2.00$ \\
B & $15.00 \pm 1.73$ & $11.67 \pm 1.53$ & $9.33 \pm 1.15$ & $6.00 \pm 1.00$ & $0.00 \pm 0.00$ & $0.00 \pm 0.00$ \\
C & $20.67 \pm 2.08$ & $18.67 \pm 1.53$ & $16.33 \pm 0.58$ & $13.67 \pm 1.53$ & $10.33 \pm 1.15$ & $7.67 \pm 2.52$ \\
D & $16.67 \pm 1.53$ & $13.00 \pm 1.73$ & $10.00 \pm 2.65$ & $0.00 \pm 0.00$ & $0.00 \pm 0.00$ & $0.00 \pm 0.00$ \\
E & $18.33 \pm 0.58$ & $16.00 \pm 1.00$ & $14.67 \pm 1.53$ & $11.33 \pm 1.15$ & $8.67 \pm 2.08$ & $0.00 \pm 0.00$ \\
F & $15.67 \pm 1,15$ & $13.33 \pm 1.53$ & $10,67 \pm 0.58$ & $10.00 \pm 1.00$ & $8.33 . \pm 0.58$ & $5.33 \pm 1.53$ \\
G & $19.33 \pm 1.53$ & $16.00 \pm 2.00$ & $13.67 \pm 2.08$ & $13.00 \pm 100$ & $9.33 \pm 0.58$ & $0.00 \pm 0.00$ \\
H & $11.67 \pm 2.08$ & $8.67 \pm 1.15$ & $6.33 \pm 0.58$ & $0.00 \pm 0.00$ & $0.00 \pm 0.00$ & $0.00 \pm 0.00$ \\
I & $0.00 \pm 0.00$ & $0.00 \pm 0.00$ & $0.00 \pm 0.00$ & $0.00 \pm 0.00$ & $0.00 \pm 0.00$ & $0.00 \pm 0.00$ \\
J & $0.00 \pm 0.00$ & $0.00 \pm 0.00$ & $0.00 \pm 0.00$ & $0.00 \pm 0.00$ & $0.00 \pm 0.00$ & $0.00 \pm 0.00$ \\
Control & $0.00 \pm 0.00$ & $0.00 \pm 0.00$ & $0.00 \pm 0.00$ & $0.00 \pm 0.00$ & $0.00 \pm 0.00$ & $0.00 \pm 0.00$ \\
\hline
\end{tabular}

FS: Full strength. No significant difference: $(P=<0.001)$.

Control: Sterile filter disc.

Table 6. The Mean Diameter of Inhibition Zones $(\mathrm{mm})$ of Anti-microbil Activity of Dentifrice Formulations against Candida albicans

\begin{tabular}{lllllll}
$\begin{array}{l}\text { Dentifrice } \\
\text { Formulation }\end{array}$ & FS & $1: 1$ Dilution & $1: 2$ Dilution & $1: 4$ Dilution & $1: 8$ Dilution & $\begin{array}{l}1: 16 \\
\text { Dilution }\end{array}$ \\
\hline A & $18.67 \pm 0.58$ & $16.33 \pm 1.15$ & $14.00 \pm 1.00$ & $12.00 \pm 1.73$ & $9.33 \pm 1.15$ & $0.00 \pm 0.00$ \\
B & $19.00 \pm 1.00$ & $16.67 \pm 1.15$ & $13.33 \pm 2.08$ & $12.00 \pm 1.73$ & $11.67 \pm 0.58$ & $9.67 \pm 1.53$ \\
C & $16.33 \pm 1.53$ & $0.00 \pm 0.00$ & $0.00 \pm 0.00$ & $0.00 \pm 0.00$ & $0.00 \pm 0.00$ & $0.00 \pm 0.00$ \\
D & $21.33 \pm 1.15$ & $19.00 \pm 1.00$ & $17.00 \pm 1.73$ & $15.33 \pm 1.53$ & $9.67 \pm 0.58$ & $0.00 \pm 0.00$ \\
E & $21.33 \pm 1.53$ & $17.67 \pm 0.58$ & $15.00 \pm 100$ & $13.67 \pm 1.53$ & $10.00 \pm 2.00$ & $0.00 \pm 0.00$ \\
F & $22.00 \pm 1.73$ & $19.67 \pm 1.53$ & $18.00 \pm 1,00$ & $13.67 \pm 1.53$ & $10.00 \pm 2.00$ & $0.00 \pm 0.00$ \\
G & $18.00 \pm 0.00$ & $14.00 \pm 100$ & $10.33 \pm 0.58$ & $0.00 \pm 0.00$ & $0.00 \pm 0.00$ & $0.00 \pm 0.00$ \\
H & $18.67 \pm 1.15$ & $16.67 \pm 0.58$ & $14.00 \pm 1.00$ & $11.67 \pm 1.53$ & $9.33 \pm 2.08$ & $0.00 \pm 0.00$ \\
I & $0.00 \pm 0.00$ & $0.00 \pm 0.00$ & $0.00 \pm 0.00$ & $0.00 \pm 0.00$ & $0.00 \pm 0.00$ & $0.00 \pm 0.00$ \\
J & $18.67 \pm 0.58$ & $12.33 \pm 2.08$ & $0.00 \pm 0.00$ & $0.00 \pm 0.00$ & $0.00 \pm 0.00$ & $0.00 \pm 0.00$ \\
Control & $0.00 \pm 0.00$ & $0.00 \pm 0.00$ & $0.00 \pm 0.00$ & $0.00 \pm 0.00$ & $0.00 \pm 0.00$ & $0.00 \pm 0.00$
\end{tabular}

FS: Full strength. $\quad$ No significant difference: $(P=0.002)$.

Control: Sterile filter disc.

From the Table 7, it could be observed that fluoride concentration ranged between $5.1-132.4 \mathrm{mg} / \mathrm{l}$, dentifrice formulation I had the highest fluoride concentrations of $132.4 \mathrm{mg} / \mathrm{l}$ and dentifrice formulation $\mathrm{J}$ had the least concentration of $5.1 \mathrm{mg} / \mathrm{l}$. Sodium concentration ranged between 350.6$420.7 \mathrm{mg} / \mathrm{l}$, dentifrice $\mathrm{G}$ had the highest concentration of $420.7 \mathrm{mg} / \mathrm{l}$ while dentifrice formulation $D$ had the least concentration of $350.6 \mathrm{mg} / \mathrm{l}$. The concentration of sulphate ranged between $0.3-0.9 \mathrm{~g}$, dentifrice formulation $\mathrm{J}$ had the highest concentration of $0.9 \mathrm{~g}$ and formulation $\mathrm{F}$ had the least concentration of $0.3 \mathrm{~g}$. The concentration of phosphate ranged between $45.9-191.8 \mathrm{mg} / \mathrm{l}$ dentifrices $\mathrm{C}$ had the highest concentration value of $191.8 \mathrm{mg} / \mathrm{l}$ and dentifrice D had the least concentration value. Calcium and carbonate concentrations ranged between $14.7 \mathrm{mg} / \mathrm{l}$ and $8.2 \mathrm{mg} / \mathrm{l}$ - $16.1 \mathrm{mg} / \mathrm{l}$ and $12.8 \mathrm{mg} / \mathrm{l}$ respectively. Dentifrice D had the highest concentrations of $16.1 \mathrm{mg} / \mathrm{l}$ and $12.8 \mathrm{mg} / \mathrm{l}$ of calcium and carbonate while dentifrice I had the least of $14.7 \mathrm{mg} / \mathrm{l}$ and $8.2 \mathrm{mg} / \mathrm{l}$ of calcium and carbonate respectively. Chloride present in formulation $\mathrm{E}$ and $\mathrm{J}$ had the concentration of $5.6 \mathrm{mg} / \mathrm{I}$ and $5.1 \mathrm{mg} / \mathrm{l}$ respectively. Potassium and nitrate are present in formulation A only. Potassium had the concentration of $3.2 \mathrm{mg} / \mathrm{l}$ and nitrate had $11.0 \mathrm{mg} / \mathrm{l}$ concentration. And zinc was present only in formulation B with concentration of $6.2 \mathrm{mg} / \mathrm{l}$. 
Bajopas Volume 10 Number 1 June, 2017

Table 7 Elemental Composition of the Various Brands of Dentifrices Used in the Study ( $\mathrm{mg} / \mathrm{L} \mathrm{or} \mathrm{g}$ )

\begin{tabular}{lllllllllll}
\hline Dentifrice & Fluoride & Sodium & Sulphate & Phosphate & Calcium & Carbonate & Chloride & Potassium & Nitrite & Zinc \\
\hline A & 119.3 & 409.1 & NF & NF & NF & NF & NF & 3.2 & 11 & NF \\
B & 111.4 & 416.5 & 0.6 & NF & NF & NF & NF & NF & NF & 6.2 \\
C & 115.4 & 406.5 & 0.7 & 191.8 & NF & NF & NF & NF & NF & NF \\
D & 105.9 & 350.6 & 0.4 & 45.9 & 16.1 & 12.8 & NF & NF & NF & NF \\
E & 8.3 & 418.2 & 0.8 & NF & NF & NF & 5.6 & NF & NF & NF \\
F & 121.1 & 360.2 & 0.3 & NF & 14.8 & 8.8 & NF & NF & NF & NF \\
G & 111.4 & 420.7 & 0.8 & 178.5 & NF & NF & NF & NF & NF & NF \\
H & 99.6 & 378.9 & 0.5 & 87.9 & NF & NF & NF & NF & NF & NF \\
I & 104.7 & 372.6 & NF & 109.4 & 14.7 & 8.2 & NF & NF & NF & NF \\
J & 132.3 & 418.8 & 0.9 & 88.3 & NF & NF & 5.1 & NF & NF & NF \\
\hline \multicolumn{2}{r}{ NF: NOT FOUND } & & & & & & & &
\end{tabular}

\section{DISCUSSION}

The microorganisms isolated from the oral mucosa with no clinical features of dental caries were $S$. mutans, $S$. aureus, E. coli, $K$. specie and $C$. albicans. This agrees with the research work conducted by Chinenye and Emeka (2001) on antimicrobial properties of Terminalca glaucescens and Zanthoylum zanthoxyloides extract and spectrum of their activity. The present study demonstrates that the $S$. mutans among the isolated organisms in oral mucosa was found to be $30.38 \%$ which is similar to the result obtained from other studies. This agree with result obtained by Maripandi et al. (2011) on Prevalence of dental caries bacterial pathogens and evaluation of inhibitory concentration effect on different tooth pastes against Streptococcus spp. This is because of there is extensive evidence associated $S$. mutans with dental caries. It has been suggested that oral cavity may also constitute an addional and possibly more stable reservoir of respiratory pathogens (Sumi et al., 2006). S. aureus was the second most occurrence isolates with $29.11 \%$ this agrees with the work of Daniluk et al. (2006) on aerobic bacteria in the oral cavity with $S$. aureus having $30.0 \%$. Klebsiella specie and E.coli was relatively lower $12.66 \%$ and $10.13 \%$ compared with gram positive pathogens. The reason of this was very possible because Gram positive pathogens are dominant in human oral cavity. From this study C. albicans was found to be $17.77 \%$ and this agrees with the study on prevalence of dental caries bacterial pathogens by Maripandi et al. (2011). However, slight modification of the host defense system, or host ecological environment, can assist the transformation of $C$. albicans into a pathogen capable of causing infections that may be lethal.

Dentifrice A was observed to be the most effective, based on the mean diameter of the zone of produced by the dentifrice in agar disc diffusion method, against almost all the tested microorganisms. This is due to the presence of triclosan in its formulation. Manupati (2011), also showed similar result with this research as triclosan containing dentifrice are the most effective against his tested organisms. Many studies using triclosan as an anti plaque agent were carried out and have given good result (Kjaerheim and Waaler, 1994). Dentifrice formulations B, C and D are fluorinated products. Among the dentifrices, formulations $E, F$ and $G$ were obtained to be moderately effective and contain active ingredients as sodium mono fluoride, sodium fluoride and sodium lauryl sulfate products. This agrees with Manupati, (2011) where he reported antimicrobial efficacy of different toothpaste and mouth rinse. Dentifrice formulations $\mathrm{H}, \mathrm{I}$ and $\mathrm{J}$ are the least effective dentifrice as shown from the zone of inhibition measured. This is due to the presentence of potassium nitrate and sodium fluoride as active ingredient in their formulation and they lack antimicrobial activity (Kamal et al., 2010), where as formulation $\mathrm{J}$ exhibited activity on only two tested microorganisms and is also a fluorinated product, but this could be due to the fact that effectiveness of fluoride toothpaste is concentration dependent (Fejerskov and Kidd, 2003). In the present study, the herbal formulations $D$ and $G$ appeared to be equally effective as the fluoride formulations. This agrees with the work of Amrutesh et al, 2010 on Clinical Evaluation of a Novel Herbal Dental Cream.

The activity, potency, and commercial acceptability of dentifrice are associated with the fluoride and phosphate levels (Borissova, et al., 1993). Fluoride is widely known to prevent tooth decay and many dental diseases, and phosphate believed to give strong teeth because of its known association with bone formation and sustenance (Jiz et al., 1996). The fluoride level in the dentifrice is within $8.3-132.3 \mathrm{mg} / \mathrm{L}$ (Table 7). The phosphate level is present in samples $B, D, G, H, I$, and $\mathrm{J}$ where sample $\mathrm{C}$ have the highest level of phosphate $191.81 \mathrm{mg} / \mathrm{L}$. This agrees with the study of Oyewale (2005) on estimation of essential inorganic constituents in commercial toothpaste. The dentifrice with high level of phosphate will be beneficial to users who are deficient in phosphorous especially at early stages of bone development (Oyewale, 2005). 
Dentifrices D, F, and I has calcium carbonate, bone and teeth are of essentially made up calcium, the main of abrasion agent in most toothpastes is calcium carbonate this also agrees with Oyewale (2005). Dentifrice $D$ has the highest calcium, and also has phosphate a combination that could be useful for people prone to osteoporosis, a bone disorder associated with deficiency in these elements (Oyewale, 2005). The elements sodium and potassium which are present in formulation $A$, and sodium in all the formulations, with no significant role in toothpaste formulation and tooth care, are introduced into toothpastes as vehicles for incorporating fluoride, phosphate and other additives (Oyewale, 2005). Nitrate is present only in formulation $\mathrm{A}$ as potassium nitrate, Pereira and Chava (2001) revealed that $3 \%$ potassium nitrate appears to have therapeutic potential to alleviate dental hypersensitivity. Zinc and sulphate are present in formulation B in form of zinc sulfate, Navada and Kumari (2008), proved that a significant improvement in oral malodor was achieved with toothpaste containing $0.2 \%$ zinc sulphate after a single brushing.

\section{Conclusion}

Based on the result of this work $S$. mutans had highest frequency of $30.38 \%$ among pathogenic microorganisms associated with dental cavity. E. coli on the other hand had the least percentage of $10.13 \%$. The work revealed that dentifrices containing triclosan were more effective in inhibiting the growth of these microorganisms, compared to dentifrice containing sodium fluoride and plant extracts even on

\section{REFERENCES}

American Dental Association (2014). Top 10 Common Dental Problems.

Amrutesh, S.O., Malini, J.P., Tandur, P.S. and Patki, P.S. (2010). Clinical evaluation of a novel herbal dental cream in plague formation: a double-blind, randomized, controlled clinical trial. Journal of Experimental Pharmacology, 2(1): $105-109$.

Borissova, R., Debouki, A. and Nikolov, T. (1993). Titrimetric determination of phosphate and monofluorophosphate in toothpaste. Fresenium journal of analytical chemistry. 347: 63-66.

Cheesbrough, M. (2004). District Laboratory Practice in tropical countries, part 1. Second Edition. Cambridge University Press, UK. Pp 64-72.

Chinenye G and Emeka K (2001). The antimicrobial properties of terminalca glaucescens and zanthoxylum zanthoxyloides extracts and the spectrum of their activity South Americacam journal of public health 3(3) 1 11.

Clarke, J. K. (1924). The bacterial factor in the etiology of dental caries. British Journal Experimental pathology, 5: 141-147.

Daniluk, T. Fiedomk, K., Sciepuk, M., Marimba, M. L, Rozkiewicz, D., Crick - rokicka, D., Tokajuk, G. krdra, B. A, Angelika I , Stokowska W, gorska M, and kedra B .R (2006) Aerobic baeteria in the oral cauity of patients with
Klebsiella spp. which exhibited high resistance to most tested dentifrices. This suggests that the use of dentifrice made of triclosan will go a long way in controlling cases of oral contaminants.

\section{Recommendations}

On the basis of aforementioned, the following recommendations are imperative:

1. Dentifrice with antimicrobial active ingredients (Triclosan) should be used because it showed antimicrobial effectiveness in many researches including present study.

2. At least tooth brush with dentifrice twice in a day is recommended in order to reduce microbial accumulation in buccal cavity.

\section{Contribution of Authors}

This work was a collaboration effort between the authors. Author Yahaya, S. designed the study and properly organized the manuscript. Author Ramatu, A.M. managed and handled all the experimental processes. All authors read to their satisfaction and approved the final manuscript.

\section{Conflict of Interest}

There was no any complicit of interest between the authors.

\section{Acknowledgement}

The authors sincerely acknowledged the effort and contribution of staff of the Department of Microbiology, Bayero University, Kano, Nigeria and the effort of staff in the Department of Medical Microbiology Unit of Aminu Kano Teaching Hospital, Kano, Nigeria.

removable dentures. Journal of advance in medical scdenee 51 (1) 87 - 90.

Fejerskov, O. O. and Kidd, E. O. (2003). Dental caries, the disease and it's clinical management. Ist Edition.Willey-black well publishers, London.

Gamboa, F. Estupinan, M. and Galindo, A. (2004). Presence of Streptococcus mutans in saliva and its relationship with dental caries. Antimicrobial Susceptibility of the Isolates. Universitas Scientiarum, 9(2): 23-27.

Jensena, J. and Barkvol,I P. (1998). Clinical Implications of the Dry Mouth: Oral Mucosal Diseases. Annals of the New York Academy of Science. 842(1): 156-162.

Jiz, Q., Li, Y. H., Huag, J. F. and Lai, C. Q. (1996). Fluorometric determination of fluoride with 7-[(4-methyl-2-arsenophenol)azo]-8hydroxy-5-quinoline sulfonic acid. Fenxi Huaxue, 24(58): 555-558.

Kamal R. A., Radhika J. Chetan S. (2010). The antimicrobial potential of ten often used mouthwashes against four dental caries pathogens. Jundishapur journal of microbiology, 3(1): 15-27.

Kjaerhein, V. and Waaler, S. M. (1994). Experiment with triclosan containing mouthrinses: dose response andan attempt to locate the receptor site(s) of triclosan in the mouth. Advance Dental Research, 8(2):302-6. 
Manupati, P. (2011). Antimicrobial Efficacy of Different Toothpastes and Mouth rinses An invitro study. Journal of Dental Research, 8(2):85-94.

Marinho, V.C.C., Higgins, J. P. T., Sheiham, A. and Logan, S. (2009). One topical fl uoride (toothpastes, or mouthrinses, or gels, or varnishes) versus another for preventing dental caries in children and adolescents (Review). Cochrane Database System Review 1:1-15.

Maripandi, A., Arun, K. T.and Al Salamah, A. (2011). Prevalence of Dental Caries Bacterial Pathogens and Evaluation of Inhibitory Concentration Effect on Different Tooth pastes Against Streptococcus spp African Journal of Microbiology Reseach. 5(14):1778-1783.

Navada, R. and Kumari, H., Le, S., Zhang, J. (2008). Oral Malodor Reduction From a Zinc Containing Toothpaste. Journal of clinical dent.19 (2):69-73..

Oyewale, A. O. (2005). Estimation of the essential inorganic constituents of commercial toothpastes. Journal of scientific and industrial research, 1(64): 101-107.

Oztan, M. D., Kiyan, N. and Gereeker, D. (2006). Antimicrobial effect, in vitro of gutta-percha points containing root canal medications against yeasts and Enterococcus faecalis. Journal of Oral Surgery,Pathology and Radiological Endodology, 102(3): 410-416.

Patterson, M. J. (1996). 'Streptococcus'. Baron's Medical Microbiology. $4^{\text {th }}$ Edition. University of Taxas Medical Branch. ISBN-0-9631172-11.

Pereira, R. and Chava, V. K. (2001). Efficacy of $3 \%$ potasium nitrate desensitization mouthwash in the treatment of dentinal hypersensitivity. Journal of periodontal 72(12): 1720-5.

Sadeghi, M. and Assar, S. (2009). An in vitro antimicrobial activity of ten Iranian-made toothpastes. Journal of dental research, 6(2): 87-92.

Sumi $Y$, Miura nagaya $M$, Michiwaki $Y$, Uematsu $H$, (2006). Colonization on the tonyue surface by respiratony pathos fens in residents of a nursiy hone - a pilot shely . $J$. of gerontowgy 23 : 55 -5 9 .

Suave, G. Rajendra, B. M. and Seethe, K. N. (2013). A simple Melton of genomic DNA excretion from human sapless for PCR - RFLP analysis. Journal of bimolecular technology. 24(4): $224-231$. 